\title{
Correlation of Communication Skills for Emotional Empathy and Academic Achievement on Clinical Performance Examinations
}

\author{
Seon Suk Jang ${ }^{1,2}$, Ji Hyun Seo ${ }^{2,3}$, Gyeong Jae Cho ${ }^{1,4}$, Soon Chan Hong ${ }^{2,5}$ and Hyang Ok Woo ${ }^{2,3}$ \\ ${ }^{1}$ Biomedical Center (BK21), Departments of ${ }^{2}$ Medical Education, ${ }^{3}$ Pediatrics, ${ }^{4}$ Anatomy, and ${ }^{5}$ Surgery, \\ School of Medicine, Gyeongsang National University, Jinju, Korea
}

\section{정서적 공감을 위한 의사소통 기술과 임상수행평가 - 학업성취도와의 관계: 일개 의학전문대학원 자료를 중심으로}

경상대학교 ${ }^{1}$ 의생명과학사업단(BK21), 의학전문대학원 ${ }^{2}$ 의학교육학교실, ${ }^{3}$ 소아청소년과학교실, ${ }^{4}$ 해부학교실, ${ }^{5}$ 외과학교실

\section{장선숙 ${ }^{1,2}$, 서지현,3, 조경제 ${ }^{1,4}$, 홍순찬,5, 우향옥,3}

Purpose: The purpose of this study was to identify the correlation between communication skills for emotional empathy and academic achievement on the Clinical Performance Examination (CPX).

Methods: One hundred twelve medical school students were observed to determine the extent to which they applied communication skills for emotional empathy (preparation stage: interview attitude, respect; rapport stage: encouragement, active listening, will for support; empathy stage: verbal expression empathy, nonverbal expression empathy, acceptance) to the CPX, as well as their level of understanding of these skills to calculate the Pearson $r$, which can be used to determine the correlation between communication skills and academic achievement (hematochezia, fatigue, abnormal menstruation, chest pain, alcohol problems).

Results: Male students had higher scores than females for all communicational skills except verbal expression empathy. Fourth-year students had statistically more significant correlations than third-year students with regard to the rapport stage 'active listening' and empathy stage 'nonverbal expression' and abnormal menstruation and chest pain. Correlations were also more significant for hematochezia in the preparation stage 'interview attitude,' rapport stage 'encouragement,' and empathy stages 'verbal and nonverbal expression' and 'acceptance.' The empathy stage 'nonverbal expressions' was more significant for fourth-year students with alcohol problems. Third-year students largely had negative correlations between emotional empathy communication skills and CPX academic achievement, especially between the preparation stage 'respect' and abnormal menstruation, and between the rapport stage 'encouragement' and hematochezia.

Conclusion: There was a significant correlation between hematochezia, wherein MS students deliver bad news to patients, and communication skills for emotional empathy.

Key Words: Communication, Emotions, Empathy, Clinical competence

Received: January 11, 2010 - Revised: March 11, 2010 • Accepted: April 1, 2010 Corresponding Author: Seonsuk Jang Department of Medical Education, Gyeongsang National University School of Medicine, 92 Chilam-dong, Jinju 660-751, Korea

TEL) 055-751-8848 FAX) 055-751-8811 E-mail) seonsukj@hanmail.net

* Seonsuk Jang was supported by BK21 Program.
Korean J Med Educ 2010 Jun; 22(2): 121-130. doi: $10.3946 /$ kjme.2010.22.2.121.

pISSN: 2005-727X elSSN: 2005-7288

(C) The Korean Society of Medical Education. All rights reserved. 


\section{서론}

환자들은 누구나 좋은 의사를 만나고 싶어 한다. 그렇기 때 문에 환자나 주변 가족들은 짧거나 긴 시간을 투자하여 주변 사람들에게 수소문(譭闻聞)해서라도 좋은 의사를 만나려는 탐 색 시간을 가지며, 만날 의사에 대해 인맥을 동원하여 정보를 수집하고 친밀한 가운데 자신을 맡기려고 노력한다. 어떤 환 자들은 의사선생님의 권위적인 태도로 인하여 진료의 세심한 부분까지 질문하기가 어렵고 불친절하거나 무뚝뚝해서 편안 하지 않다[1]는 큰 불만도 있지만, 환자와 의사 관계의 원활한 의사소통을 위해서 좋은 의사가 갖추어야 할 핵심 역량 중 하 나는 '환자의 입장에서 아픔을 공감(empathy)'해 주는 것이다 [2]. 공감이란 상대방의 경험, 감정, 사고, 신념 등을 상대의 입장이나 관점에서 듣고 이해하는 것이다[3].

그러나 공감은 모호한 개념[4]이기 때문에 공감이라는 정 의에 대한 의견일치 부족에도 불구하고 문헌에서는 공감에 대한 다양한 해설과 특징을 부여하기도 한다[5]. 이 개념상의 모호성으로 공감은 정의하고 측정하기 어려운 개념으로 기술 되어 왔으며[6], 일반적으로 공감에 대한 연구자들은 '공감'을 인지적 속성으로 보기도 하고[7,8], 정서적인 특징으로 보기 도 하지만[9,10], 모두를 다 포함하기도 한다[11,12]. 그러나 공감이 인지적인 사고든 감정이든 상관없이 의료치료에 중요 한 요소임이 틀림없고[13] 환자에 대한 의사의 정서적 공감은 동정이 아닌 환자의 고통과 아픔을 함께 느끼는 것을 말한다. 하지만 공감이 훈련 기간을 연장하더라도 향상이 쉽지 않기 때문에[14] 우리나라 상황에서 재해석하여 교육과정에 반영 할 의사소통 기술이 필요하며[15], 의사가 환자의 입장에서 짧은 시간 안에 효율적인 의사소통을 위해서는 준비단계, 신 뢰단계, 공감단계 등 세 과정으로 나누어 다음과 같은 정서적 공감을 위한 의사소통 기술들을 제안해야 한다. 환자가 의사 와 상호작용할 수 있는 단계는 준비단계, 신뢰단계, 공감단계 로 나눌 수 있으며, 의사가 환자를 위하여 세 단계에서 정서적 공감 표현을 할 수 있는 의사소통 기술은 다음과 같다.

준비단계

첫째, 환자를 보면 '나는 어떤 태도로 대할 것인가라는 스 스로 물음을 갖는다(면담태도[16]).
둘째, 환자에 대한 존중감을 가지고 대면 준비를 한다(존중 [17]).

\section{신뢰단계}

셋째, 환자가 아픔에도 불구하고 병원을 내방한 것에 대해 격려한다(격려[18]).

넷째, 의사가 환자병력에 적극적인 경청의 자세를 표현한 다(적극적 경청[18]).

다섯째, 의사가 환자를 진심으로 돕고자 한다는 의지를 표 현한다(돕고자하는 의지).

공감단계

여섯째, 환자의 언어적 표현에 공감을 한다(언어표현 공감 [19]).

일곱째, 환자의 아픈 표정이나 태도, 몸짓 등 비언어적인 표 현에 공감을 한다(비언어표현 공감[19]).

여덟째, 환자의 아픔이 어느 정도인지를 감지했다는 수용 의 표현을 한다(수용).

본 연구는 정서적 공감의 의사소통 기술이 제일 중요하게 다루어지는 임상수행평가(clinical performance examination, $\mathrm{CPX}$ )에서 의학전문대학원 학생들이 공감을 위한 의사 소통 기술에 대하여 어느 정도 인식하여 실행하고 있는지, 공 감적 의사소통기술과 학업성취도와의 관계는 어떠한지를 분 석하고자 한다.

\section{대상 및 방법}

연구대상은 경남 소재 G 대학 3, 4학년으로 2009년 3월 초 부터 7월 초까지 $\mathrm{CPX}$ 시험 후 설문지를 배포하여 회수하는 방식으로 연구를 진행하였다. 총 정원 159 명 중 지속적인 설 문에 불성실하게 답하거나 무응답 문항을 남긴 응답지를 제 외하고 112 명을 최종 분석대상으로 하였다(Table 1).

정서적 공감을 위한 의사소통 기술은 준비단계의 면담태도, 존중, 신뢰단계의 격려, 적극적 경청, 돕고자하는 의지, 공감 단계의 언어표현 공감, 비언어표현 공감, 수용 등으로 각각 두 문항씩 16 문항으로 구성되어 있다. 문항의 내용으로는 $\mathrm{CPX}$ 에서 기술을 어느 정도로 실시하였는지, 기술을 어느 정도 알 고 있는지의 질문에 Likert식 9점 척도로 '전혀 그렇지 않다' 
의 1점에서부터 '매우 그렇다'의 9점으로 반응하여 점수가 높 을수록 정서적 공감을 위한 의사소통 기술이 높은 것으로 해 석하였다. 또한, 학업성취도는 3학년(2009년 7월 11일)과 4학 년(2009년 6월 11일)의 공통된 시험내용으로 이루어진 총 5 문항(혈변, 피로, 월경이상, 가슴통증, 음주문제)이다.

자료는 SPSS windows version 14.0 (SPSS Inc., Chicago, USA)을 이용하여 분석하였다. 학생들의 개인적 특성에 따라 정서적 공감을 위한 의사소통 기술과 $\mathrm{CPX}$ 학업성취도의 차 이가 있는지 알아보기 위해 t-test와 일원배치 분산분석을 실 시하였고(Tables 2, 3), 정서적 공감을 위한 의사소통 기술과 $\mathrm{CPX}$ 학업성취도 간의 상호상관을 살펴보기 위해서는 Pearson의 적률상관계수(Pearson r)를 구하여 비교하였다 (Tables 4, 5, 6, 7, 8).

\section{결과}

\section{1. 연구대상자들의 일반적인 특성}

연구 대상자의 특성을 보면(Table 1), 총 연구 대상자는 112 명으로 3학년이 62명(55.4\%), 4학년이 50명(44.6\%)으로 유의수준 0.05 에서 성별과 연령, 성별과 대학 전공계열 간에 통계적으로 유의한 차이가 있었다. 학생들의 연령은 26세에 서 30세까지 77명(68\%)으로 가장 많았다. 성별 분포를 보면 남학생은 61명, 여학생은 51명이었다. 남학생은 26세 30세 (60.7\%)와 31세 35세(32.8\%), 여학생은 거의 26세 30세가 대부분이었다(78.4\%). 대학전공계열은 남학생이 생물학계열 자연과학(41.0\%)과 공학계열(39.3\%)로 많았으며, 여학생은 생물학계열 자연과학(40.2\%), 공학계열(26.8\%)과 비생물학 계열 자연과학 $(16.1 \%)$ 등의 순으로 많았다.

\section{2. 정서적 공감을 위한 의사소통 기술}

학생들의 일반적인 특성(성별, 학년, 연령, 대학전공계열)에 따른 공감을 위한 의사소통기술들(준비단계: 면담태도, 존중; 신뢰단계: 격려, 적극적 경청, 돕고자하는 의지; 공감단계: 언 어표현 공감, 비언어표현 공감, 수용)의 독립표본 $\mathrm{t}$-검증과 일 원배치 분산분석을 실시한 결과는 다음과 같다(Table 2).
Table 1. General Characteristics of Medical School students

\begin{tabular}{lccccc}
\hline \multirow{2}{*}{ Item } & \multicolumn{2}{c}{ Gender } & Total & $\begin{array}{c}x^{2} \\
(\mathrm{p})\end{array}$ \\
\cline { 2 - 3 } $\begin{array}{lccccc}\text { Grade } \\
3\end{array}$ & $35(57.4)$ & $27(52.9)$ & $62(55.4)$ & 0.220 \\
4 & $26(42.6)$ & $24(47.1)$ & $50(44.6)$ & $(0.638)$ \\
Age & & & & \\
Blow 26 & $1(1.6)$ & $5(9.8)$ & $6(5.4)$ & \\
$26 \sim 30$ & $37(60.7)$ & $40(78.4)$ & $77(68.8)$ & $12.529^{\text {a) }}$ \\
$31 \sim 35$ & $20(32.8)$ & $6(11.8)$ & $26(23.2)$ & $(0.006)$ \\
Above 35 & $3(4.9)$ & $0(0.0)$ & $3(2.7)$ & \\
Degree type & & & & \\
Biology & $25(41.0)$ & $20(39.2)$ & $45(40.2)$ & \\
Nonbiology & $7(11.5)$ & $11(21.6)$ & $18(16.1)$ & $16.253^{\mathrm{a})}$ \\
Engineering & $24(39.3)$ & $6(11.8)$ & $30(26.8)$ & $(0.003)$ \\
Paramedic & $3(4.9)$ & $11(21.6)$ & $14(12.5)$ & \\
LHMS & $2(3.3)$ & $3(5.9)$ & $5(4.5)$ & \\
Total & $61(100.0)$ & $51(100.0)$ & $112(100.0)$ & \\
\hline
\end{tabular}

Values are presented as number $(\%)$.

Nonbiology: Nonbiology Natural Sciences, Paramedic: Veterinary, Nursing \& Pharmaceutical Sciences, LHMS: Literature, Humanities, Management \& Social Sciences.

a) $p<0.01$.

첫째, 준비단계의 면담태도 $(\mathrm{t}=3.389, \mathrm{p}=0.001)$ 와 존중 $(\mathrm{t}=3.391, \mathrm{p}=0.001)$ 은 남학생이 여학생보다 높은 점수를 나타 냈다. 둘째, 신뢰단계의 격려 $(\mathrm{t}=2.823, \mathrm{p}=0.006)$, 적극적 경청 $(\mathrm{t}=2.823, \mathrm{p}=0.006)$ 그리고 돕고자하는 의지 $(\mathrm{t}=2.800, \mathrm{p}=$ 0.006)도 남학생이 여학생보다 높은 점수를 보였으며, 적극적 경청 $(\mathrm{t}=-2.150, \mathrm{p}=0.034)$ 은 4학년이 3학년보다 높은 점수를 나타냈다. 셋째, 공감단계의 언어표현 공감은 차이를 보이는 항목이 없었으나, 비언어표현 공감 $(\mathrm{t}=2.334, \mathrm{p}=0.021)$ 과 수용 $(\mathrm{t}=2.761, \mathrm{p}=0.007)$ 은 역시 남학생이 여학생에 비하여 의미있 는 차이를 보였으며, 비언어적 표현 공감 $(\mathrm{t}=-2.738, \mathrm{p}=0.007)$ 은 3학년보다 4학년의 점수가 높은 것으로 나타났다.

\section{3. $\mathrm{CPX}$ 학업성취도}

학생들의 일반적인 특성(성별, 학년, 연령, 대학전공계열) 에 따른 $\mathrm{CPX}$ 학업성취도(혈변, 피로, 월경이상, 가슴통증, 음 주문제)의 독립표본 $\mathrm{t}$-검증과 일원배치 분산분석을 실시한 결과는 다음과 같다(Table 3).

첫째, 혈변과 피로문항은 학생들의 일반적인 특성에서 차 이를 보이지 않았다. 둘째, 월경이상문항 $(\mathrm{t}=-4.237, \mathrm{p}=0.000)$ 
Seonsuk Jang, et al: Correlation of Communication Skills for Emotional Empathy and Academic Achievement on Clinical Performance Examinations

Table 2. Comparison of Communication Skills for Emotional Empathy according to Medical School student characteristics

\begin{tabular}{|c|c|c|c|c|c|c|c|c|c|c|c|c|c|c|c|c|}
\hline \multirow{3}{*}{ Item } & \multicolumn{4}{|c|}{ Preparation stage } & \multicolumn{6}{|c|}{ Rapport stage } & \multicolumn{6}{|c|}{ Empathy stage } \\
\hline & \multicolumn{2}{|c|}{$\begin{array}{l}\text { Interview } \\
\text { attitude }\end{array}$} & \multicolumn{2}{|c|}{ Respect } & \multicolumn{2}{|c|}{ Encouragement } & \multicolumn{2}{|c|}{$\begin{array}{l}\text { Active } \\
\text { listening }\end{array}$} & \multicolumn{2}{|c|}{$\begin{array}{l}\text { Will for } \\
\text { support }\end{array}$} & \multicolumn{2}{|c|}{$\begin{array}{c}\text { Verbal } \\
\text { expression } \\
\text { empathy }\end{array}$} & \multicolumn{2}{|c|}{$\begin{array}{c}\text { Nonverbal } \\
\text { expression } \\
\text { empathy }\end{array}$} & \multicolumn{2}{|c|}{ Acceptance } \\
\hline & $\mathrm{M}$ & SD & M & SD & $\mathrm{M}$ & SD & $\mathrm{M}$ & SD & $\mathrm{M}$ & SD & $\mathrm{M}$ & SD & $\mathrm{M}$ & SD & $\mathrm{M}$ & SD \\
\hline \multicolumn{17}{|l|}{$\begin{array}{l}\text { Gender } \\
\end{array}$} \\
\hline Male & 11.16 & 2.37 & 12.59 & 2.57 & 11.16 & 2.21 & 11.98 & 2.09 & 10.84 & 2.28 & 11.51 & 2.75 & 11.15 & 2.82 & 11.61 & 2.60 \\
\hline Female & 9.65 & 2.35 & 10.90 & 2.69 & 9.88 & 2.60 & 10.51 & 2.21 & 9.47 & 2.79 & 10.61 & 2.55 & 9.92 & 2.71 & 10.24 & 2.64 \\
\hline$t(p)$ & $3.389^{b}$ & ' & $3.391^{b /}$ & $(0.001)$ & $2.823^{b /}$ & $(0.006)$ & $3.615^{b l}$ & (0.000) & $2.800^{b l}$ & $(0.006)$ & 1.785 & (0.077) & $2.334^{a}$ & $(0.021)$ & $2.761^{b /}$ & $(0.007)$ \\
\hline \multicolumn{17}{|l|}{ Age } \\
\hline Blow 30 & 10.28 & 2.45 & 11.82 & 2.77 & 10.46 & 2.51 & 11.18 & 2.23 & 10.11 & 2.79 & 11.01 & 2.80 & 10.48 & 2.94 & 11.01 & 2.71 \\
\hline Above 31 & 11.03 & 2.47 & 11.83 & 2.73 & 10.93 & 2.36 & 11.69 & 2.35 & 10.52 & 1.98 & 11.34 & 2.35 & 10.90 & 2.48 & 10.90 & 2.70 \\
\hline$t(p)$ & -1.430 & (0.156) & -0.014 & $(0.989)$ & -0.889 & (0.376) & -1.043 & (0.299) & -0.855 & (0.395) & -0.573 & (0.568) & -0.679 & $(0.499)$ & 0.198 & (0.844) \\
\hline \multicolumn{17}{|l|}{ Grade } \\
\hline 3 & 10.08 & 2.65 & 11.58 & 2.78 & 10.29 & 2.47 & 10.92 & 2.51 & 9.89 & 2.82 & 10.81 & 2.83 & 9.95 & 2.94 & 10.60 & 2.78 \\
\hline 4 & 10.96 & 2.15 & 12.12 & 2.70 & 10.94 & 2.44 & 11.80 & 1.82 & 10.62 & 2.28 & 11.46 & 2.47 & 11.38 & 2.47 & 11.46 & 2.53 \\
\hline$t(p)$ & -1.897 & $(0.061)$ & -1.034 & (0.303) & -1.392 & $(0.167)$ & $-2.150^{a \mid}$ & $(0.034)$ & -1.489 & $(0.139)$ & -1.285 & $(0.202)$ & $-2.738^{b}$ & $(0.007)$ & -1.700 & (0.092) \\
\hline \multicolumn{17}{|l|}{ Degree type } \\
\hline Biology & 10.24 & 2.36 & 12.04 & 2.40 & 10.47 & 2.49 & 11.22 & 2.10 & 10.00 & 2.76 & 10.67 & 2.81 & 10.38 & 2.94 & 11.09 & 2.57 \\
\hline Nonbiology & 9.72 & 2.63 & 11.28 & 3.34 & 10.22 & 2.86 & 10.56 & 2.57 & 10.17 & 2.94 & 10.67 & 2.87 & 10.00 & 3.24 & 10.33 & 3.29 \\
\hline Engineering & 11.00 & 2.56 & 12.03 & 2.68 & 10.93 & 2.03 & 11.97 & 1.92 & 10.67 & 2.11 & 11.60 & 2.37 & 11.23 & 2.47 & 11.10 & 2.59 \\
\hline Paramedic & 10.36 & 2.27 & 10.57 & 3.13 & 10.21 & 2.83 & 10.57 & 2.79 & 9.71 & 3.05 & 10.93 & 2.46 & 10.07 & 2.56 & 10.71 & 2.55 \\
\hline$F(p)$ & 1.118 & $(0.346)$ & 1.306 & $(0.276)$ & 0.440 & (0.725) & 2.038 & (0.113) & $0.546 \mid$ & $(0.652)$ & 0.833 & $0.479)$ & 0.998 & $(0.397)$ & 0.412 & (0.745) \\
\hline
\end{tabular}

Degree type: LHMS whose frequency is less than 10 was excluded.

${ }^{a)}<0.05,{ }^{b \mid} p<0.01$.

Table 3. Comparison of CPX achievement according to MS student characteristics

\begin{tabular}{|c|c|c|c|c|c|c|c|c|c|c|}
\hline \multirow[t]{2}{*}{ Item } & \multicolumn{2}{|c|}{ Hematochezia } & \multicolumn{2}{|c|}{ Fatigue } & \multicolumn{2}{|c|}{$\begin{array}{c}\text { Abnormal } \\
\text { menstruation }\end{array}$} & \multicolumn{2}{|c|}{ Chest pain } & \multicolumn{2}{|c|}{$\begin{array}{l}\text { Alcohol } \\
\text { problem }\end{array}$} \\
\hline & $\mathrm{M}$ & SD & $\bar{M}$ & SD & $\mathrm{M}$ & SD & $\bar{M}$ & SD & $\mathrm{M}$ & SD \\
\hline \multicolumn{11}{|l|}{ Gender } \\
\hline Male & 60.37 & 10.66 & 54.24 & 7.92 & 60.73 & 11.16 & 66.10 & 9.01 & 37.82 & 13.21 \\
\hline Female & 62.24 & 11.86 & 56.90 & 7.95 & 60.97 & 10.56 & 68.08 & 8.27 & 43.14 & 11.44 \\
\hline$t(p)$ & \multicolumn{2}{|c|}{$-0.879(0.382)$} & \multicolumn{2}{|c|}{$-1.765(0.080)$} & \multicolumn{2}{|c|}{$-0.117(0.907)$} & \multicolumn{2}{|c|}{$-1.200(0.233)$} & \multicolumn{2}{|c|}{$-2.252^{\text {al) }}(0.026)$} \\
\hline \multicolumn{11}{|l|}{ Age } \\
\hline Blow 30 & 61.55 & 11.50 & 55.94 & 7.35 & 60.85 & 10.51 & 67.96 & 8.53 & 40.81 & 12.67 \\
\hline Above 31 & 60.28 & 10.47 & 54.06 & 9.67 & 60.82 & 11.94 & 64.26 & 8.72 & 38.62 & 12.73 \\
\hline$t(p)$ & \multicolumn{2}{|c|}{$0.523(0.602)$} & \multicolumn{2}{|c|}{$1.088(0.279)$} & \multicolumn{2}{|c|}{0.014 (0.989) } & \multicolumn{2}{|c|}{$2.002^{a /}(0.048)$} & \multicolumn{2}{|c|}{$0.800(0.425)$} \\
\hline \multicolumn{11}{|l|}{ Grade } \\
\hline 3 & 61.42 & 14.11 & 54.45 & 8.45 & 57.21 & 10.26 & 63.21 & 8.68 & 38.11 & 13.93 \\
\hline 4 & 60.98 & 6.06 & 56.69 & 7.33 & 65.34 & 9.89 & 71.70 & 6.07 & 42.88 & 10.42 \\
\hline$t(p)$ & \multicolumn{2}{|c|}{$0.220(0.826)$} & \multicolumn{2}{|c|}{$-1.501(0.136)$} & \multicolumn{2}{|c|}{$-4.237^{b /}(0.000)$} & \multicolumn{2}{|c|}{$-6.077^{b /}(0.000)$} & \multicolumn{2}{|c|}{$-2.009^{\text {al }}(0.047)$} \\
\hline \multicolumn{11}{|l|}{ Degree type } \\
\hline Biology & 61.51 & 9.92 & 55.47 & 7.70 & 61.07 & 10.90 & 67.28 & 7.76 & 39.49 & 11.45 \\
\hline Nonbiology & 59.62 & 11.86 & 52.79 & 8.97 & 58.41 & 8.10 & 69.85 & 10.71 & 39.63 & 9.76 \\
\hline Engineering & 63.51 & 9.12 & 55.25 & 8.66 & 60.27 & 11.79 & 64.75 & 9.45 & 39.48 & 13.80 \\
\hline Paramedic & 62.07 & 14.65 & 58.77 & 5.66 & \multirow{2}{*}{\multicolumn{2}{|c|}{$\begin{array}{cc}67.69 & 7.09 \\
2.352 & (0.077)\end{array}$}} & \multirow{2}{*}{\multicolumn{2}{|c|}{$\begin{array}{cc}67.86 & 6.55 \\
1.380 & (0.253)\end{array}$}} & \multirow{2}{*}{\multicolumn{2}{|c|}{$\begin{array}{cc}46.49 & 17.38 \\
1.209 & (0.310)\end{array}$}} \\
\hline$F(p)$ & \multicolumn{2}{|c|}{$0.512(0.675)$} & \multicolumn{2}{|c|}{$1476(0.225)$} & & & & & & \\
\hline
\end{tabular}

Degree type: LHMS whose frequency is less than 10 was excluded.

CPX: clinical performance examination.

${ }^{a)} \mathrm{p}<0.05,{ }^{\text {b) }} \mathrm{p}<0.01$. 
은 4학년이 3학년보다 높은 점수를 나타냈다. 셋째, 가슴통증 문항은 나이와 학년에서 차이를 보였는데 나이는 30세 이하 가 31세 이상에 비하여 점수가 높은 것으로 나타났으며 $(\mathrm{t}=$ 2.002, $\mathrm{p}=0.048)$, 학년은 4학년이 3학년에 비하여 점수가 높 은 것으로 나타났다 $(\mathrm{t}=-6.077, \mathrm{p}=0.000)$. 넷째, 음주문제문항 은 성별과 학년에서 차이를 보였는데 성별은 여자가 남학생 에 비하여 점수가 높은 것으로 나타났고 $(\mathrm{t}=-2.252, \mathrm{p}=0.026)$, 학년은 4학년이 3학년보다 높은 점수를 보였다 $(\mathrm{t}=-2.009$, $\mathrm{p}=0.047$ ).

\section{4. 정서적 공감을 위한 의사소통 기술과 $\mathrm{CPX}$ 학업 성취도 간 상관관계}

정서적 공감을 위한 의사소통 기술(준비단계: 면담태도, 존 중; 신뢰단계: 격려, 적극적 경청, 돕고자하는 의지; 공감단 계: 언어표현 공감, 비언어표현 공감, 수용)과 학업성취도(혈 변, 피로, 월경이상, 가슴통증, 음주문제)의 관계(Table 4)가 연구대상자의 일반적 특성인 성별(Table 5), 학년(Table 6), 연령(Table 7), 대학전공계열(Table 8)에 따라 차이가 있는지 알아보기 위한 결과는 다음과 같다.

Table 4. Correlation between Communication Skills for Emotional Empathy and CPX Achievement

\begin{tabular}{|c|c|c|c|c|c|c|}
\hline \multirow{2}{*}{\multicolumn{2}{|c|}{ Communication skills for emotional empathy }} & \multicolumn{5}{|c|}{ Name of subject } \\
\hline & & \multirow{2}{*}{$\begin{array}{c}\text { Hematochezia } \\
-0.020\end{array}$} & \multirow{2}{*}{$\begin{array}{c}\text { Fatigue } \\
-0.023\end{array}$} & \multirow{2}{*}{$\begin{array}{c}\begin{array}{c}\text { Abnormal } \\
\text { menstruation }\end{array} \\
0.191^{\mathrm{al}}\end{array}$} & \multirow{2}{*}{$\begin{array}{l}\text { Chest } \\
\text { pain } \\
-0.045\end{array}$} & \multirow{2}{*}{$\begin{array}{r}\begin{array}{r}\text { Alcohol } \\
\text { problem }\end{array} \\
0.104\end{array}$} \\
\hline Preparation & Interview attitude & & & & & \\
\hline stage & Respect & -0.074 & -0.053 & -0.040 & 0.037 & -0.022 \\
\hline Rapport & Encouragement & -0.122 & -0.073 & 0.035 & 0.035 & -0.054 \\
\hline \multirow[t]{2}{*}{ stage } & Active listening & -0.069 & -0.022 & 0.033 & 0.028 & -0.009 \\
\hline & Will for support & -0.009 & -0.011 & 0.025 & -0.060 & 0.036 \\
\hline & Empathy for verbal expression & -0.083 & -0.062 & 0.062 & -0.003 & 0.049 \\
\hline \multirow{2}{*}{$\begin{array}{l}\text { Empatny } \\
\text { stage }\end{array}$} & Empathy for nonverbal expression & -0.109 & -0.025 & 0.114 & 0.101 & 0.072 \\
\hline & Acceptance & -0.046 & 0.054 & 0.122 & 0.078 & 0.023 \\
\hline
\end{tabular}

CPX: clinical performance examination.

${ }^{a)}<0.05$.

Table 5. Correlation of Communication Skills for Emotional Empathy and CPX Achievement according to Gender

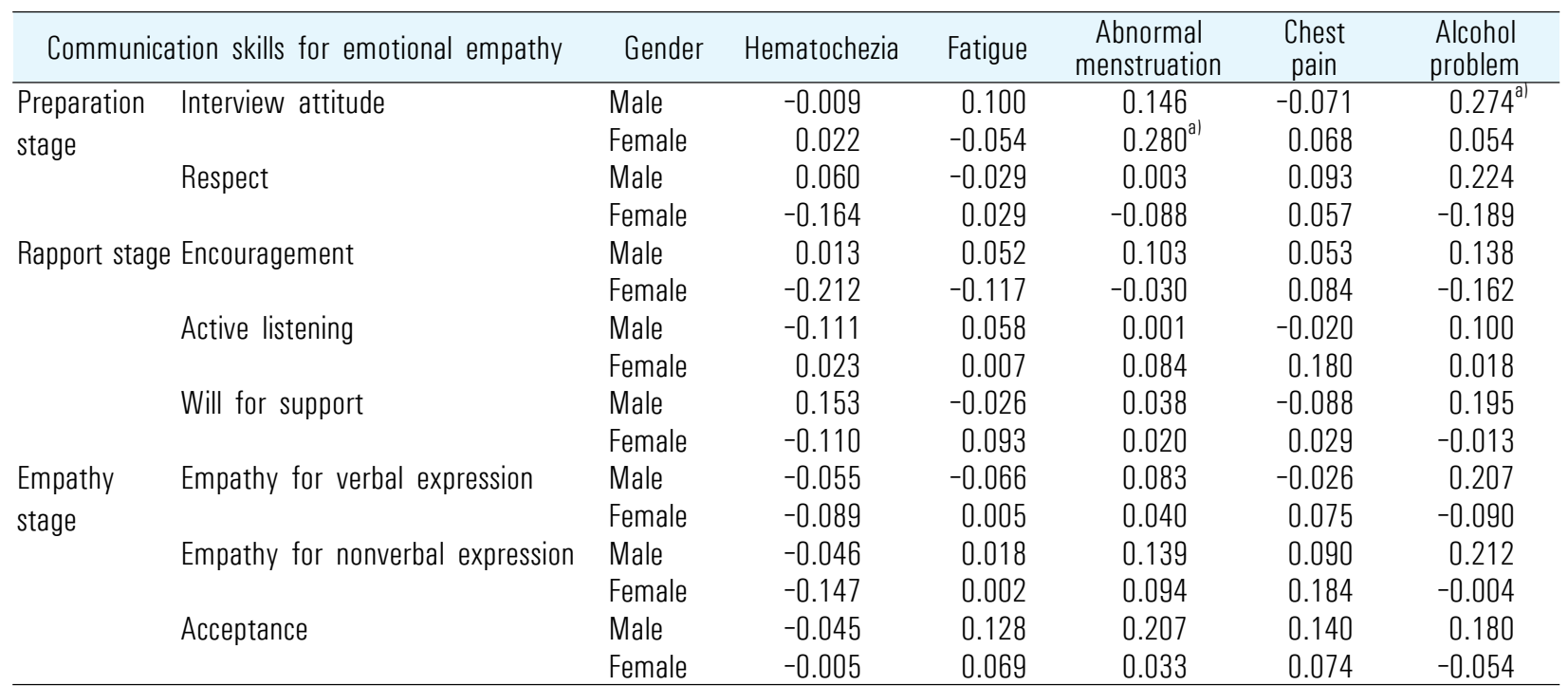

CPX: clinical performance examination.

${ }^{a)}<0.05$. 
첫째, 정서적 공감을 위한 의사소통 기술과 학업성취도 간 의 관계를 분석하기 위하여 상관분석을 실시한 결과, 준비단 계의 면담태도와 월경이상문항에서 정적상관 $(\mathrm{r}=0.191)$ 으로 면담태도 점수가 높을수록 월경이상에 대한 학업성취도 점수 가 높아진다고 할 수 있다(Table 4).
둘째, 성별에 따른 정서적 공감을 위한 의사소통 기술과 학 업성취도 간의 상관분석을 실시한 결과, 남학생은 준비단계의 면담태도와 음주문제문항에 정적상관 $(r=0.274)$ 이 있는 것으 로 나타났으며, 여학생은 준비단계의 면담태도와 월경이상문 항에서 정적상관 $(\mathrm{r}=0.280)$ 이 있는 것으로 나타났다(Table 5).

Table 6. Correlation of Communication Skills for Emotional Empathy and CPX Achievement according to Grade

\begin{tabular}{|c|c|c|c|c|c|c|c|}
\hline \multicolumn{2}{|c|}{ Communication skills for emotional empathy } & \multirow{2}{*}{$\begin{array}{c}\text { Grade } \\
3\end{array}$} & \multirow{2}{*}{$\begin{array}{c}\text { Hematochezia } \\
-0.116\end{array}$} & \multirow{2}{*}{$\begin{array}{l}\text { Fatigue } \\
-0.065\end{array}$} & \multirow{2}{*}{$\begin{array}{c}\begin{array}{c}\text { Abnormal } \\
\text { menstruation }\end{array} \\
0.017\end{array}$} & \multirow{2}{*}{$\begin{array}{c}\begin{array}{c}\text { Chest } \\
\text { pain }\end{array} \\
-0.162\end{array}$} & \multirow{2}{*}{$\begin{array}{c}\text { Alcohol } \\
\text { problem } \\
-0.001\end{array}$} \\
\hline Preparation & Interview attitude & & & & & & \\
\hline \multirow[t]{3}{*}{ stage } & & 4 & $0.335^{\mathrm{al}}$ & -0.019 & $0.328^{\mathrm{al}}$ & -0.136 & 0.227 \\
\hline & Respect & 3 & -0.173 & -0.168 & $-0.255^{\mathrm{al}}$ & -0.023 & -0.087 \\
\hline & & 4 & 0.210 & 0.081 & 0.146 & 0.008 & 0.036 \\
\hline Rapport & Encouragement & 3 & $-0.292^{\mathrm{al}}$ & -0.188 & -0.167 & 0.013 & -0.212 \\
\hline \multirow[t]{5}{*}{ stage } & & 4 & $0.352^{\mathrm{al}}$ & 0.045 & 0.182 & -0.121 & 0.137 \\
\hline & Active listening & 3 & -0.144 & -0.100 & -0.161 & -0.084 & -0.124 \\
\hline & & 4 & 0.237 & 0.045 & 0.164 & -0.062 & 0.129 \\
\hline & Will for support & 3 & -0.086 & -0.141 & -0.178 & -0.150 & -0.040 \\
\hline & & 4 & 0.278 & 0.164 & 0.205 & -0.146 & 0.111 \\
\hline \multirow{6}{*}{$\begin{array}{l}\text { Empathy } \\
\text { stage }\end{array}$} & Empathy for verbal expression & 3 & -0.198 & -0.123 & -0.144 & -0.110 & -0.078 \\
\hline & & 4 & $0.292^{\text {al }}$ & -0.010 & 0.259 & 0.004 & 0.229 \\
\hline & Empathy for nonverbal expression & 3 & -0.227 & -0.159 & -0.113 & -0.066 & -0.107 \\
\hline & & 4 & $0.286^{\mathrm{al}}$ & 0.100 & 0.230 & 0.057 & $0.289^{\mathrm{al}}$ \\
\hline & Acceptance & 3 & -0.186 & -0.052 & -0.056 & 0.025 & -0.102 \\
\hline & & 4 & $0.401^{\mathrm{bl}}$ & 0.164 & 0.245 & -0.049 & 0.165 \\
\hline
\end{tabular}

CPX: clinical performance examination.

${ }^{a)}<0.05,{ }^{b)} p<0.01$.

Table 7. Correlation of Communication Skills for Emotional Empathy and CPX Achievements according to Age

\begin{tabular}{|c|c|c|c|c|c|c|c|}
\hline \multicolumn{2}{|c|}{ Communication skills for emotional empathy } & Age & Hematochezia & Fatigue & $\begin{array}{c}\text { Abnormal } \\
\text { menstruation }\end{array}$ & $\begin{array}{l}\text { Chest } \\
\text { pain }\end{array}$ & $\begin{array}{l}\text { Alcohol } \\
\text { problem }\end{array}$ \\
\hline Preparation & \multirow[t]{2}{*}{ Interview attitude } & Blow 30 & -0.069 & 0.021 & 0.138 & -0.056 & 0.026 \\
\hline \multirow[t]{3}{*}{ stage } & & Above 31 & 0.163 & -0.075 & 0.336 & 0.079 & $0.377^{\text {al }}$ \\
\hline & \multirow[t]{2}{*}{ Respect } & Blow 30 & -0.183 & -0.005 & -0.099 & 0.078 & -0.186 \\
\hline & & Above 31 & 0.284 & -0.164 & 0.114 & -0.077 & $0.464^{\mathrm{al}}$ \\
\hline \multirow{6}{*}{$\begin{array}{l}\text { Rapport } \\
\text { stage }\end{array}$} & \multirow{2}{*}{ Encouragement } & Blow 30 & -0.188 & -0.118 & -0.041 & 0.103 & -0.126 \\
\hline & & Above 31 & 0.119 & 0.059 & 0.243 & -0.106 & 0.193 \\
\hline & \multirow[t]{2}{*}{ Active listening } & Blow 30 & -0.139 & -0.035 & 0.013 & -0.009 & -0.051 \\
\hline & & Above 31 & 0.163 & 0.035 & 0.082 & 0.202 & 0.136 \\
\hline & \multirow[t]{2}{*}{ Will for support } & Blow 30 & -0.082 & 0.007 & -0.001 & 0.036 & 0.024 \\
\hline & & Above 31 & 0.342 & -0.039 & 0.122 & $-0.390^{a)}$ & 0.112 \\
\hline Empathy & \multirow[t]{2}{*}{ Empathy for verbal expression } & Blow 30 & -0.147 & -0.069 & 0.015 & -0.009 & -0.040 \\
\hline \multirow[t]{5}{*}{ stage } & & Above 31 & 0.177 & -0.027 & 0.210 & 0.060 & $0.380^{\mathrm{al}}$ \\
\hline & \multirow{2}{*}{ Empathy for nonverbal expression } & Blow 30 & -0.213 & -0.053 & 0.106 & 0.177 & -0.006 \\
\hline & & Above 31 & 0.301 & 0.069 & 0.145 & -0.093 & $0.368^{\mathrm{al}}$ \\
\hline & \multirow[t]{2}{*}{ Acceptance } & Blow 30 & -0.093 & 0.038 & -0.006 & 0.121 & -0.075 \\
\hline & & Above 31 & 0.101 & 0.085 & $0.454^{\mathrm{al}}$ & -0.057 & 0.304 \\
\hline
\end{tabular}

CPX: clinical performance examination.

${ }^{\text {a) }} \mathrm{p}<0.05$. 
셋째, 학년에 따른 정서적 공감을 위한 의사소통 기술과 학 업성취도의 상관분석을 실시한 결과, 준비단계의 면담태도는 4학년에서 혈변문항 $(\mathrm{r}=0.335)$ 과 월경이상문항 $(\mathrm{r}=0.328)$ 에서 정적상관이 있는 것으로 나타났으며, 존중은 3학년에서 '월경 이상과 부적상관 $(\mathrm{r}=-0.255)$ 이 있는 것으로 나타났다. 신뢰단 계의 격려는 3학년에서 혈변문항과 부적상관 $(\mathrm{r}=-0.292)$ 이 있 는 것으로 나타났으나, 4학년에서는 '혈변'과 정적상관 $(\mathrm{r}=$ 0.352)이 있는 것으로 나타났다. 공감단계의 언어표현 공감은 4 학년에서 혈변문항과 정적상관 $(\mathrm{r}=0.292)$ 이 있는 것으로 나 타났다. 비언어표현 공감은 4학년에서 혈변문항( $\mathrm{r}=0.286)$ 과 음주문제문항 $(\mathrm{r}=0.289)$ 과 정적상관이 있는 것으로 나타났으 며, 수용은 4학년에서 혈변문항과 정적상관 $(\mathrm{r}=0.401)$ 이 있는
것으로 나타났다(Table 6).

넷째, 연령에 따른 공감을 위한 의사소통 기술과 학업성취 도 간의 상관분석을 실시한 결과 30세 이하는 상관이 있는 항 목이 없는 것으로 나타났다. 그러나, 31세 이상은 준비단계의 면담태도에서 음주문제문항과 정적상관 $(\mathrm{r}=0.377)$ 이 있는 것 으로 나타났으며, 존중은 음주문제문항과 정적상관 $(\mathrm{r}=0.464)$ 이 있는 것으로 나타났다. 신뢰단계의 돕고자 하는 의지는 가 슴통증문항과 부적상관 $(\mathrm{r}=-0.390)$ 이 있는 것으로 나타났으 며, 공감단계의 언어표현 공감은 음주문제문항과 정적상관 $(\mathrm{r}=0.380)$ 이 있는 것으로 나타났다. 비언어표현 공감은 음주 문제문항과 정적상관 $(\mathrm{r}=0.368)$ 이 있는 것으로 나타났으며, 수용은 월경이상문항과 정적상관 $(\mathrm{r}=0.454)$ 이 있는 것으로 나

Table 8. Correlation of Communication Skills for Emotional Empathy and CPX Achievement according to Degree Type

\begin{tabular}{|c|c|c|c|c|c|c|c|}
\hline \multicolumn{2}{|c|}{ Communication skills for emotional empathy } & \multirow{2}{*}{$\begin{array}{l}\text { Degree type } \\
\text { Biology }\end{array}$} & Hematochezia & \multirow{2}{*}{$\begin{array}{r}\text { Fatigue } \\
0.133\end{array}$} & \multirow{2}{*}{$\begin{array}{c}\begin{array}{c}\text { Abnormal } \\
\text { menstruation }\end{array} \\
0.228\end{array}$} & \multirow{2}{*}{$\begin{array}{c}\begin{array}{c}\text { Chest } \\
\text { pain }\end{array} \\
0.008\end{array}$} & \multirow{2}{*}{$\begin{array}{r}\begin{array}{r}\text { Alcohol } \\
\text { problem }\end{array} \\
0.041\end{array}$} \\
\hline Preparation & Interview attitude & & -0.005 & & & & \\
\hline \multirow[t]{7}{*}{ stage } & & Nonbiology & 0.195 & 0.426 & 0.262 & -0.012 & $0.600^{b /}$ \\
\hline & & Engineering & -0.184 & $-0.370^{a l}$ & 0.277 & 0.055 & 0.088 \\
\hline & & Paramedic & 0.252 & $-0.647^{a)}$ & 0.455 & -0.008 & 0.001 \\
\hline & Respect & Biology & -0.159 & 0.043 & -0.140 & 0.026 & $-0.311^{\text {al }}$ \\
\hline & & Nonbiology & 0.170 & 0.453 & 0.127 & 0.224 & $0.570^{\mathrm{al}}$ \\
\hline & & Engineering & 0.084 & $-0.398^{\mathrm{al}}$ & 0.145 & 0.095 & $0.373^{\text {al }}$ \\
\hline & & Paramedic & -0.173 & -0.407 & 0.126 & -0.149 & -0.360 \\
\hline Rapport & Encouragement & Biology & -0.053 & 0.086 & 0.002 & 0.045 & -0.284 \\
\hline \multirow[t]{11}{*}{ stage } & & Nonbiology & 0.047 & 0.221 & 0.238 & 0.270 & $0.636^{b l}$ \\
\hline & & Engineering & -0.051 & $-0.398^{\mathrm{al}}$ & 0.105 & -0.025 & 0.089 \\
\hline & & Paramedic & -0.279 & -0.339 & -0.051 & -0.003 & -0.189 \\
\hline & Active listening & Biology & -0.230 & 0.078 & -0.090 & 0.017 & -0.225 \\
\hline & & Nonbiology & 0.305 & 0.396 & 0.283 & 0.370 & $0.650^{\mathrm{bl}}$ \\
\hline & & Engineering & -0.059 & -0.240 & 0.110 & 0.004 & 0.104 \\
\hline & & Paramedic & 0.009 & $-0.591^{\mathrm{al}}$ & $0.562^{a 1}$ & 0.036 & -0.064 \\
\hline & Will for support & Biology & -0.114 & 0.117 & 0.009 & 0.006 & 0.023 \\
\hline & & Nonbiology & 0.204 & 0.359 & 0.223 & 0.057 & $0.584^{\mathrm{al}}$ \\
\hline & & Engineering & 0.102 & $-0.436^{\mathrm{al}}$ & 0.076 & -0.219 & -0.052 \\
\hline & & Paramedic & -0.039 & -0.194 & 0.079 & -0.020 & -0.148 \\
\hline \multirow{12}{*}{$\begin{array}{l}\text { Empathy } \\
\text { stage }\end{array}$} & Empathy for verbal expression & Biology & -0.012 & -0.051 & 0.082 & -0.006 & -0.188 \\
\hline & & Nonbiology & 0.227 & 0.386 & 0.295 & 0.081 & $0.674^{\mathrm{bl}}$ \\
\hline & & Engineering & -0.066 & -0.329 & 0.054 & 0.013 & 0.213 \\
\hline & & Paramedic & -0.254 & -0.346 & 0.238 & 0.253 & 0.031 \\
\hline & Empathy for nonverbal expression & Biology & -0.129 & 0.016 & 0.159 & 0.150 & -0.183 \\
\hline & & Nonbiology & 0.122 & 0.283 & 0.339 & 0.282 & $0.809^{\mathrm{bl}}$ \\
\hline & & Engineering & -0.065 & -0.167 & 0.029 & 0.048 & 0.229 \\
\hline & & Paramedic & -0.168 & -0.403 & 0.342 & 0.221 & -0.023 \\
\hline & Acceptance & Biology & -0.113 & 0.063 & -0.055 & 0.011 & -0.208 \\
\hline & & Nonbiology & 0.271 & $0.556^{\mathrm{a})}$ & 0.289 & 0.293 & $0.604^{\mathrm{bl}}$ \\
\hline & & Engineering & -0.027 & -0.170 & $0.468^{b /}$ & 0.168 & 0.150 \\
\hline & & Paramedic & -0.023 & -0.412 & 0.032 & 0.006 & -0.144 \\
\hline
\end{tabular}

Degree type: LHMS whose frequency is less than 10 was excluded.

CPX: clinical performance examination.

${ }^{\text {a) }}<0.05,{ }^{\text {b) }} \mathrm{p}<0.01$. 
타났다(Table 7).

다섯째, 대학전공계열에 따른 정서적 공감을 위한 의사소 통 기술과 학업성취도 간의 상관분석을 실시한 결과는 다음 과 같다. 준비단계의 면담태도는 비생물학계열 자연과학과 음주문제문항에서 정적상관 $(\mathrm{r}=0.600)$, 공학계열은 '피로'와 부적상관 $(\mathrm{r}=-0.370)$, 의학계열 역시 피로문항과 부적상관 $(\mathrm{r}=-0.647)$ 이 있는 것으로 나타났으며, 존중은 생물학계열 자 연과학과 음주문제문항에서 부적상관( $\mathrm{r}=-0.311)$, 비생물학계 열 자연과학은 음주문제문항과 정적상관 $(\mathrm{r}=0.570)$ 이 있는 것 으로 나타났고, 공학계열은 피로문항과 부적상관( $\mathrm{r}=-0.398)$, 음주문제문항과 정적상관 $(\mathrm{r}=0.373)$ 이 있는 것으로 나타났다. 신뢰단계의 격려는 비생물계열 자연과학과 음주문제문항에 서 정적상관 $(\mathrm{r}=0.636)$, 공학계열은 피로문항과 부적상관 $(\mathrm{r}=$ -0.398), 적극적 경청은 비생물계열 자연과학과 음주문제문 항에서 정적상관 $(\mathrm{r}=0.650)$ 이 있는 것으로 나타났으며, 의학 계열은 피로문항과 부적상관 $(\mathrm{r}=-0.591)$, 월경이상문항과 정 적상관 $(\mathrm{r}=0.562)$ 이 있는 것으로 나타났다. 돕고자하는 의지 는 비생물계열 자연과학과 음주문제문항에서 정적상관 $(\mathrm{r}=$ $0.584)$, 공학계열은 피로문항과 부적상관(r=-0.436)이 있는 것으로 나타났다. 공감단계의 언어표현 공감은 비생물계열 자연과학과 음주문제문항에서 정적상관 $(\mathrm{r}=0.674)$, 비언어표 현 공감은 비생물계열 자연과학과 음주문제문항에서 정적상 관 $(\mathrm{r}=0.809)$, 수용은 비생물계열 자연과학과 피로문항 $(\mathrm{r}=$ $0.558)$, 음주문제문항 $(\mathrm{r}=0.604)$ 에서 정적상관이 있는 것으로 나타났으며, 공학계열은 월경이상문항과 정적상관 $(\mathrm{r}=0.468)$ 이 있는 것으로 나타났다(Table 8).

\section{고찰}

본 연구는 의학전문대학원 3, 4학년 학생들이 CPX시험에 서 표준화환자(standardized patient)들에게 정서적 공감을 위한 의사소통기술(준비단계: 면담태도, 존중; 신뢰단계: 격 려, 적극적 경청, 돕고자하는 의지; 공감단계: 언어표현 공감, 비언어표현 공감, 수용)을 어느 정도 인식하는 가운데 실제 적용하였는지를 알아보고 이러한 의사소통기술과 CPX 학업 성취도(혈변, 피로, 월경이상, 가슴통증, 음주문제) 간의 상관
관계를 살펴보았으며 통계적으로 유의미한 결과와 후속 연구 에 대한 제언은 다음과 같다.

첫째, 남학생이 여학생보다 정서적 공감을 위한 의사소통 기술이 공감단계의 언어표현 공감을 제외한 준비단계(면담태 도, 존중), 신뢰단계(격려, 적극적 경청, 돕고자하는 의지), 공 감단계(비언어표현 공감, 수용) 등에서 높은 점수를 나타냈으 며, 면담태도에서 남학생은 음주문제문항과 여학생은 월경이 상문항과 정적인 상관을 보였다는 것이다. 이러한 결과는 남 자의사보다 여자의사들이 정서에 초점을 맞추어 대화를 한다 는 결과[20]와 공감척도가 여자가 남자보다 높았다는 결과[4] 와도 상반된다. 다년간 연구에서 공감이 떨어지는 정도가 의 대 여학생보다 남학생에게서 더 컸다는 연구결과[4]를 살펴 볼 때, 우리나라 의전원이나 의대 교과과정 속에서 학년별로 체계적인 교육을 받지 못한 학생들의 공감개념 재정립과 장 기적인 연구로 어느 시점에서 상승하고 하락하는지, 성별 차 이가 발생하는 시점과 이유가 무엇인지에 대한 후속 연구가 필요하다.

둘째, 4학년이 월경이상과 가슴통증, 음주문제문항에서 3 학년보다 높은 점수를 얻은 것은 문항을 반복하여 경험한 연 습효과라 볼 수 있다. 공감을 위한 의사소통기술(신뢰단계: 적극적 경청; 공감단계: 비언어적 표현 공감)과 혈변문항(준 비단계: 면담태도, 신뢰단계의 격려; 공감단계: 언어표현 공 감, 비언어표현 공감, 수용 등) 그리고 음주문제문항(공감단 계: 비언어 표현 공감) 등의 유의미한 결과는, 나쁜 소식전하 기 등 표준화환자와의 면담술 피드백을 경험한 4학년들이 의 사-환자관계에서 정서적 공감을 위한 의사소통기술의 필요 성을 깊이 인식한 결과라 여겨진다.

셋째, 정서적 공감을 위한 의사소통기술과 연령이나 대학 전공계열, $\mathrm{CPX}$ 학업성취도와 연령, 대학전공계열과 유의미 한 점수 차이는 없었으나, 31세 이상의 학생들은 대체로 정서 적 공감을 위한 의사소통기술 점수가 높을수록 학업성취도의 음주문제문항 점수도 상승하는 경향을 보였고, 대학전공계열 이 비생물학계열 자연과학인 경우 음주문제문항과 공감을 위 한 의사소통기술의 준비단계(면담태도, 존중), 신뢰단계(격 려, 적극적 경청, 돕고자하는 의지), 공감단계(언어표현 공감, 비언어표현 공감, 수용)에서 정적상관을 나타냈다는 것이다. 이 결과, 본 연구에 참여한 학생들의 인문사회계열 빈도수가 
부족함으로 학부의 전공(인문사회계열, 자연과학계열)과 의 사-환자관계에 대한 태도 차이가 없다는 연구[21]와 비교하 기에 적절하지 못하지만, 연령과 공감능력의 유의한 관련성 이 없다는 연구결과[22]와는 상반됨으로 대학의 전공계열이 어떤 영향을 미치는지 보다 세밀한 후속 연구가 필요하다.

넷째, 3학년이 정서적 공감을 위한 의사소통기술과 $\mathrm{CPX}$ 학업성취도 간의 상관연구에서 대부분 음의 관계를 나타냈으 며 특히, 준비단계의 존중과 월경이상 그리고 신뢰단계의 격 려와 혈변에서는 의미있는 음의 상관관계를 보였다는 것은 3학년들이 의사에게 공감은 별로 필요 없는 덕목으로 여기는 것은 아닌지 염려되며, 공감점수가 의대 첫 2년간은 크게 변 하지 않았으나 3학년말에 공감점수가 유의미하게 크게 떨어 졌다는 Hojat et al.[4]의 장기연구와 일치한다. 그러나, 본 연 구의 측정도구는 정서적 공감을 위한 의사소통기술들 중의 하나인 자기공감평가 결과로서 의사-환자 관계의 충분한 공 감평가만을 반영하지 않기 때문에 직접 비교는 어렵지만, 3 학 년의 유의미한 공감 잠식 이유로는 3학년부터 시작되는 본 대 학의 임상실습과 역할모델의 부재, 공부해야 할 많은 학습자 료, 시간압박, 환자와 환경적 요인 등을 생각해 볼 수 있다. 시간이 흐를수록 컴퓨터를 기초로 하는 진단 및 치료기술에 대한 학생들의 지나친 의존이 환자와의 만남에서 인간적인 상호작용의 중요성에 대한 비전을 제한할 수도 있기 때문에 [4], 의대나 의전원 내 의생활전문상담센터를 신설하여 학생 들의 정서적 공감을 위한 의사소통 기술 향상에 힘써야 할 것 이다. 상담은 특히 3학년 학생들의 학업과 관련된 스트레스 요인들을 탐색하고 적응을 도우며, 함께 참여한 동료들을 존 중하고 참여한 용기에 격려하며 서로의 이야기에 경청함으로 써 자신을 이해하고 참여하는 동료들로부터 도움을 받을 뿐 만 아니라 자신도 동료들을 도와주는 체험기회를 부여한다. 따라서 학생들이 공감을 직접 경험하면서 개념의 변화를 느 낄 수 있는 공감증진 상담프로그램의 개발이 필요하다.

본 연구의 문제점은 일개 의학전문대학원의 학생들을 대상 으로 일회적인 분석이라는 점이다. 하지만 의대학생 $96.5 \%$ 의 학생이 면담기술을 향상시킬 필요가 있다고 답하여 면담교육 에 대한 필요성과 요구도가 높음을 알 수 있듯이[16] 주제 및 대상별 의사소통 기술을 우리나라 상황에서 재해석하여 교육 과정에 반영할 필요가 있기 때문에[15] 학생들의 공감증진을
위한 다양한 연구에 미흡하나마 작은 보탬이 되고자 한다. 본 연구 결과는 의전원 학생들이 나쁜 소식전하기 같은 심각한 결과를 설명하는 문항은 환자와 정서적 공감을 위한 의사소 통기술들과 대체로 관련됨을 인식하지만, 그 외의 문항은 정 서적 공감을 위한 의사소통기술과 관련이 없는 것으로 나타 났다. 그러나 진료를 받는 환자들은 정서적 공감을 위한 의사 소통의 모든 기술들이 의사에게 배어 있기를 기대하며, 지금 도 좋은 의사가 되고자 열심히 공부하는 의대생이나 의전원 생들에게 컴퓨터에 의한 진단이나 치료기술에 대해 지나친 믿음을 갖게 하기 보다는 환자를 만나 사람 상호 간의 관계가 제일 밑거름이 되어야 한다는 믿음을 심어주는 교육환경이 되기를 기대한다.

\section{REFERENCES}

1. Ahn S, Lee YM, Ahn DS. A study on how young doctors and patients perceive the doctor-patient relationship. Korean J Med Educ 2006; 18: 279-287.

2. Lee YM, Ahn DS. A preliminary study for exploring the attributes of being a "good doctor". Korean J Med Educ 2007; 19: 313-323.

3. Yang YS. Mediating effects of empathy and forgiveness on the relationship between marital conflicts and psychological maladjustment [dissertation]. [Seoul, Korea]: Sookmyung Women's University; 2008.

4. Hojat M, Vergare MJ, Maxwell K, Brainard G, Herrine SK, Isenberg GA, Veloski J, Gonnella JS. The devil is in the third year: a longitudinal study of erosion in empathy in medical school. Acad Med 2009; 84: 1182-1191.

5. Hojat M. Empathy in patient care: antecedents, development, measurement, and outcomes. New York, USA: Springer; 2007.

6. Kestenbaum R, Farber EA, Sroufe LA. Individual differences in empathy among preschoolers: relation to attachment history. In: Eisenberg N, editor. Empathy and related emotional responses. San Francisco, USA: 
Jossey-Bass; 1989. p. 51-64.

7. Kohut H. The analysis of the self: a systematic approach to the psychoanalytic treatment of narcissistic personality disorders. New York, USA: International Universities Press; 1971.

8. Basch MF. Empathic understanding: a review of the concept and some theoretical considerations. J Am Psychoanal Assoc 1983; 31: 101-126.

9. Eisenberg N. Empathy and related emotional responses. San Francisco, USA: Jossey-Bass; 1989.

10. Hoffman ML. The development of empathy. In: Rushton JP, Sorrentino RM, editors. Altruism and helping behavior: social, personality, and developmental perspectives. Hillsdale, USA: Lawrence Erlbaum Associates, Inc.; 1981. p. 41-63.

11. Davis MH. Measuring individual differences in empathy: evidence for a multidimensional approach. J Pers Soc Psychol 1983; 44: 113-126.

12. Hodges SD, Wegner DM. Automatic and controlled empathy. In: Ickes W, editor. Empathic accuracy. New York, USA: Guilford; 1997. p. 311-339.

13. Spiro H. Commentary: the practice of empathy. Acad Med 2009; 84: 1177-1179.

14. Kim YH, Yang JH, Ahn SY, Song SY, Roh H. Communication skills improvement of medial students according to length and methods of preclinical training.
Korean J Med Educ 2009; 21: 3-16.

15. Yang E. Teaching and learning communication skills in medical education. Korean J Med Educ 2008; 20: 99107.

16. Choi CJ, Kim JM, Park YG. Patient-centered attitudes and communication skills in medical students after clerkship. Korean J Med Educ 2004; 16: 169-177.

17. Branch WT Jr. Viewpoint: teaching respect for patients. Acad Med 2006; 81: 463-467.

18. Makoul G. Essential elements of communication in medical encounters: the Kalamazoo consensus statement. Acad Med 2001; 76: 390-393.

19. Shapiro J. How do physicians teach empathy in the primary care setting? Acad Med 2002; 77: 323-328.

20. Roter DL, Hall JA, Aoki Y. Physician gender effects in medical communication: a meta-analytic review. JAMA 2002; 288: 756-764.

21. Park J. Medical students' attitudes toward the patientdoctor relationship. Korean J Med Educ 2007; 19: 215-223.

22. Lee BK, Bahn GH, Lee WH, Park JH, Yoon TY, Baek SB. The relationship between empathy and medical education system, grades, and personality in medical college students and medical school students. Korean J Med Educ 2009; 21: 117-124. 